\title{
Visual long-term memory for spatial frequency?
}

\author{
MARTIN LAGES and AILEEN PAUL \\ University of Glasgow, Glasgow, Scotland
}

\begin{abstract}
It has been suggested that a visual long-term memory based on a sensory representation of the stimulus accounts for discrimination performance when the reference and the test stimuli are separated in time. Decision processes involved in setting response criteria, however, may also contribute to discrimination performance. In the present study, it is shown that under proper control, spatial frequency discrimination thresholds from a group of observers, each performing on a single trial, are significantly higher for a 2-h than for a 5-sec retention interval, whereas thresholds from individual observers performing in repeated trials with a 2-h retention interval are considerably lower. The results suggest that discrimination performance may depend on the retention of task-relevant information, such as a response criterion, rather than on visual memory of the stimulus. It is concluded that it is risky to postulate a high-fidelity long-term visual memory for spatial frequency on the basis of psychophysical group discrimination thresholds.
\end{abstract}

Human observers are capable of recognizing many objects and scenes over long periods of time (see, e.g., Rock \& Engelstein, 1959; Standing, 1973). On the other hand, observers fail to notice large changes in scenes after short intervals (see, e.g., Rensink, O’Regan, \& Clark, 2000). These paradoxical findings on visual memory lead to the important question of how visual information is represented in memory. Do we store sensory representations in a visual memory system, or do we retain more abstract information that is task relevant and not necessarily visual? This question also relates to arguments against the necessity of positing specific memory stores for different modalities (e.g., Cowan, 1999; Crowder, 1993; Nairne, 1990,2003 ) and to the influence of decisional factors in visual detection and discrimination (e.g., Swift \& Smith, 1983; Vogels \& Orban, 1986; Wenger \& Rasche, 2005). Although the existence of a visual long-term memory has intuitive appeal, storage and retrieval of task-relevant information as part of a decision process cannot be ruled out. In the following psychophysical study, we contrast predictions of a "perfect" visual long-term memory with entrainment of a response criterion in a delayed discrimination task.

In a two-interval forced choice task, Magnussen and Dyrnes (1994) obtained "perfect" retention of spatial frequency information for up to $50 \mathrm{~h}$, thereby extending previous findings on spatial frequency storage and retrieval from short-term to long-term memory. Lages and Treisman (1998), however, showed that spatial frequency

Parts of this research were presented at the Annual Meeting of the Vision Sciences Society in 2004. This research was supported by the Royal Society of London and EPSRC (GR/R61215/01). We thank Michel Treisman and Michael Wenger for helpful comments. Correspondence concerning this article should be addressed to M. Lages, Department of Psychology, University of Glasgow, 58 Hillhead Street, Glasgow G12 8QB, Scotland (e-mail: m.lages@psy.gla.ac.uk). discrimination data that had been thought to demonstrate visual long-term memory could be accounted for by criterion setting at the decision stage. Individual performance in a given trial was based on a comparison between the test stimulus and a decision criterion, rather than on a sensory memory of the reference stimulus. For example, spatial frequency discrimination was not impaired when observers had to guess a midpoint from a few test stimuli in the absence of a reference (Lages, 1998; Experiment 2 in Lages \& Treisman, 1998).

Signal detection theory (SDT; Green \& Swets, 1974; Wickens, 2002) attributes discrimination to a decision process that compares the incoming sensory input, as registered on the decision axis, with a response criterion whose value is determined by such parameters as signal probability and the benefits and costs of different outcomes. This contrasts with the idea of sensory memories, which attributes discrimination to a comparison of the incoming sensory input with the neural representation of a past sensory input. Criterion-setting theory is an extension of SDT that proposes that a response criterion is not constant during a session but changes in value from trial to trial under the impact of processes aimed at optimizing performance (Treisman \& Faulkner, 1985; Treisman \& Williams, 1984). These processes also provide an explanation for the occurrence of sequential dependencies between trials, which are understood not as random errors, but as an expression of the working of a criterion-setting mechanism. As in SDT, criterion setting assumes that discrimination may make use of a long-term reference criterion. But it assumes in addition that the system computes trial-to-trial adjustments of the response criterion, with the object of ensuring that the criterion on each trial is optimal for that decision.

In an attempt to separate a visual long-term memory from confounding criterion-setting processes, Magnussen, Greenlee, Aslaksen, and Kildebo (2003) conducted 
an interesting experiment in which each subject was instructed on the task with gratings presented on screen before attending a single test trial. In the test trial, a reference grating was shown, and after a retention interval of $5 \mathrm{sec}$ or $24 \mathrm{~h}$, a single test grating of lower or higher spatial frequency was presented to different subjects. Each subject judged whether the bars of the test grating were thinner or thicker than the previously seen reference grating. The responses of as many subjects as there were trials were pooled to establish a group psychometric function. They concluded, from similar slopes for a 5-sec and a 24-h interstimulus interval (ISI), that visual memory holds an accurate representation of spatial frequency over a 24-h period (see Figure 1).

A difficulty with the logic of claiming perfect visual long-term memory on the basis that group discrimination thresholds do not change as time interval increases is that this argument rests on affirming the null hypothesis and on assuming that group performance corresponds to individual performance (see, however, Ashby, Maddox, \& Lee, 1994). Note that discrimination performance with similar discrimination thresholds does not necessarily imply the same point of subjective equality (PSE). In the framework of criterion-setting theory, similar group discrimination thresholds for a prolonged ISI would be attributed to the ability of subjects to retain individual response criteria that are placed sufficiently close together on a decision axis.

One way of addressing this issue is to make sure that during instructions no feasible response criterion is estab- lished at the reference value and to compare group with individual discrimination performance in the same task. If a high-fidelity representation of spatial frequency is retained in visual long-term memory, psychometric functions fitted to individual, as well as group, data should exhibit similar discrimination thresholds or slopes, and the PSE should be centered on the same spatial frequency. If the observer has no opportunity to establish a feasible response criterion before the test trial and visual long-term memory is weak or absent, the position of the response criterion may vary considerably between individual observers, particularly in a single test trial. Thus, pooling of responses across observers would be equivalent to sampling from distributions with different means, and a measure of dispersion, such as the discrimination threshold, should be significantly higher for group data than for individual data. In addition, we would predict considerable shifts of PSEs in some individual data sets as a consequence of uncertainty about the reference.

During instructions, Magnussen et al. (2003) illustrated the task by showing a low and a high spatial frequency grating on screen, with spatial frequencies centered on the reference spatial frequency. This may have helped observers to establish a response criterion before the test trial. In the following experiment, each observer was instructed on the task using training stimuli with low and high spatial frequencies printed on paper, so that test stimuli presented on screen were centered on a different spatial frequency. This minor change was introduced to make sure that the observers in the group condition could not use a response

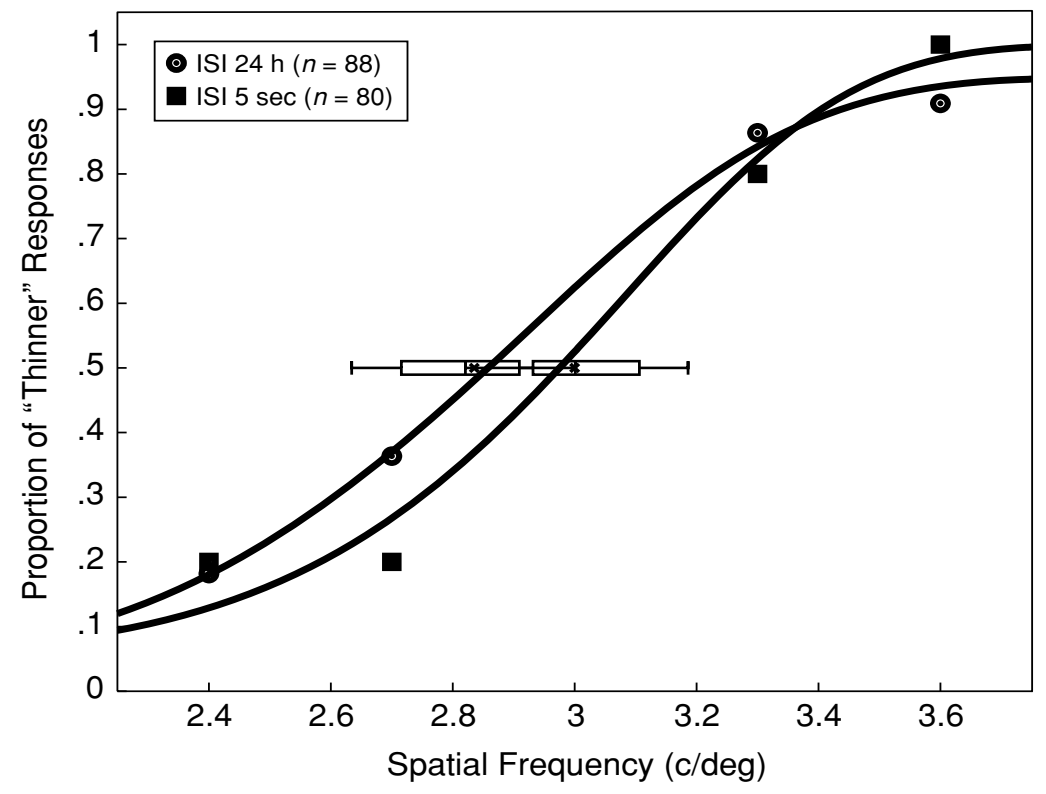

Figure 1. Weibull psychometric functions fitted to proportions of thinner responses in Magnussen, Greenlee, Aslaksen, and Kildebo's (2003) data. Circles depict group data $(n=88)$ for a retention interval of $24 \mathrm{~h}($ ISI $=24 \mathrm{~h})$, and squares depict group data $(n=80)$ for a retention interval of $5 \mathrm{sec}$ (ISI = $5 \mathrm{sec}$ ). Confidence intervals for PSE at $50 \%$ are obtained by bootstrapping (Wichmann \& Hill, 2001a, 2001b). Boxes and bars indicate Weibull confidence intervals of $68.3 \%$ and $95.3 \%$, respectively. 
criterion that had been established during training. A change of training stimuli should have no detrimental effect on visual short-term or long-term memory in a subsequent test trial. At the same time, this change is likely to increase uncertainty about the reference, because each observer views a single reference on screen, rather than a range of stimuli centered on the reference.

\section{METHOD}

Students from Glasgow University participated in a spatial frequency discrimination experiment under conditions similar to those reported in Magnussen et al. (2003). The observers were instructed on the task by viewing a low and a high spatial frequency grating printed on paper before they participated in a single test trial with different gratings displayed on screen.

\section{Subjects}

Undergraduate and graduate students from Glasgow University participated in two groups ( $n=93$ and $n=75)$. Two graduate students (R.G. and L.O.), also from Glasgow University, and one of the authors (M.L.) discriminated in a condition with repeated trials. All the subjects were naive as to the aims of the experiment, except for Observer M.L.

\section{Stimuli and Procedure}

During instructions, the observers viewed vertical sine wave gratings of $0.4 \mathrm{~cm}$ and $1.2 \mathrm{~cm}$ per cycle in a Gaussian envelope $(S D=$ $1.1 \mathrm{~cm}$ ), each printed on a sheet of A4 paper.

The stimuli on screen were also vertical sine wave gratings in a Gaussian envelope ( $S D=2^{\circ}$ visual angle) displayed for $5 \mathrm{sec}$. Images subtended $12^{\circ}$ of visual angle at a viewing distance of $57 \mathrm{~cm}$. They were shown at $30 \%$ Michelson contrast on a Sony GDM F500R 21-in. flat CRT monitor calibrated in luminance. Supported by a chin- and headrest, all the observers viewed a reference grating of $3 \mathrm{c} / \mathrm{deg}$. After a retention interval of $5 \mathrm{sec}$ (ISI $=5 \mathrm{sec}$ ) for a group of $n=75$ observers and approximately $2 \mathrm{~h}$ (ISI $=2 \mathrm{~h}$ ) for a second group of $n=93$ observers, a test stimulus was displayed that differed in spatial frequency from the $3.0 \mathrm{c} / \mathrm{deg}$ reference by $0 \%, \pm 10 \%$, or $\pm 20 \%$. Each observer had to indicate whether the bars of the test grating appeared thinner or thicker than the bars of the reference grating by pressing a labeled key. For 5-sec and 2-h ISIs, responses from single trials were pooled across observers to establish a group psychometric function. The 3 observers in the individual condition viewed test gratings monocularly through a circular aperture of $12^{\circ}$ visual angle in order to exclude the use of peripheral size cues over trials. These observers attended 20 trials on consecutive days (22-h intertrial interval [ITI]) with a retention interval of approximately $2 \mathrm{~h}$. Responses from each observer were collapsed over repeated trials to establish individual psychometric functions.

\section{Psychometric Function}

A Weibull distribution function $W(x ; \alpha, \beta)$ was fitted to each data set, using a constrained maximum likelihood fit (Wichmann \& Hill, 2001a):

$$
W(x ; \alpha, \beta)=1-\exp \left[-(x / \alpha)^{\beta}\right], 0 \leq x<\infty .
$$

The psychometric function $\Psi(x)$ is described by four parameters, where $\alpha$ corresponds to a probability of approximately .63 and $\beta$ describes the steepness of the function:

$$
\Psi(x ; \alpha, \beta, \gamma, \lambda)=\gamma+(1-\gamma-\lambda) W(x ; \alpha, \beta) .
$$

The parameters $\gamma$ and $\lambda$ are constrained to values between 0 and .05 and refer to a limited guess rate and miss rate, respectively. Confidence intervals of parameter estimates were determined by parametric bootstrapping, using a bias-corrected and accelerated method over 2,999 runs (Wichmann \& Hill, 2001b).

Derivatives of the fitted Weibull function $d W(x) / d x$ evaluated at the $25 \%, 50 \%$, and $75 \%$ points served as estimates of slope. Discrimination thresholds (Weber fractions) are expressed as $\left[\Psi^{-1}(.75)-\right.$ $\left.\Psi^{-1}(.25)\right] / 2$, with spatial frequency standardized to 1.0 arbitrary units.

\section{RESULTS}

We reanalyzed Magnussen et al.'s (2003) data and confirmed that there was no statistically significant difference between slopes of the fitted Weibull functions for 5-sec and 24-h ISIs under the present fitting regime (see Figure 1 and Table 1). Mean estimated slopes are slightly lower for the $24-\mathrm{h}$ ISI, but still within $68 \%$ confidence intervals at $25 \%, 50 \%$, and $75 \%$ thinner responses. However, the PSE of $2.83 \mathrm{c} / \mathrm{deg}$ for the 24-h ISI group is significantly shifted away from the PSE of $3.0 \mathrm{c} / \mathrm{deg}$ for the $5-\mathrm{sec}$ ISI. Note that

Table 1

Constrained Maximum Likelihood Fit of Weibull Function to Magnussen,

\begin{tabular}{|c|c|c|c|c|c|c|c|c|c|c|}
\hline \multirow[b]{2}{*}{$n$} & \multirow[b]{2}{*}{ ISI } & \multicolumn{6}{|c|}{ Weibull Fit } & \multicolumn{3}{|c|}{ Derived Measures } \\
\hline & & $\alpha$ & $\beta$ & $\gamma$ & $\lambda$ & $D$ & $p$ & PSE & Slope & Weber \\
\hline \multicolumn{11}{|c|}{ Magnussen et al. (2003) } \\
\hline 88 & $24 \mathrm{~h}$ & 2.98 & 7.50 & .01 & .05 & 0.31 & .20 & 2.83 & 0.92 & 0.098 \\
\hline 80 & $5 \mathrm{sec}$ & 3.12 & 9.31 & .05 & .00 & 2.23 & .76 & 3.00 & 1.08 & 0.084 \\
\hline \multicolumn{11}{|c|}{ Present Data } \\
\hline 93 & $2 \mathrm{~h}$ & 3.85 & 2.18 & .01 & .01 & 0.09 & .01 & 3.26 & 0.23 & 0.383 \\
\hline 75 & $5 \mathrm{sec}$ & 3.23 & 6.88 & .00 & .05 & 0.68 & .14 & 3.07 & 0.78 & 0.115 \\
\hline R.G. & $2 \mathrm{~h}$ & 2.72 & 12.4 & .01 & .00 & 0.00 & .37 & 2.64 & 1.63 & 0.055 \\
\hline L.O. & $2 \mathrm{~h}$ & 3.49 & 11.0 & .00 & .05 & 1.88 & .71 & 3.38 & 1.13 & 0.080 \\
\hline M.L. & $2 \mathrm{~h}$ & 3.15 & 12.4 & .00 & .00 & 0.58 & .55 & 3.06 & 1.40 & 0.065 \\
\hline
\end{tabular}
Greenlee, Aslaksen, and Kildebo's (2003) and the Present Data

Note-Columns denote number of observations ( $n$ ), interstimulus intervals (ISIs), four parameter estimates, and deviation of fit $D$ with probability $p$. Derived measures are the $50 \%$ point (PSE), the first derivative of the psychometric function at the $50 \%$ point (slope), and the discrimination threshold with SF standardized to 1.0 (Weber). 
"perfect" visual memory for a single spatial frequency cannot explain significant displacements of the PSE after a prolonged retention interval.

In Magnussen et al.'s (2003) study, the group discrimination threshold for a retention interval of $5 \mathrm{sec}$ was slightly lower than that in our experiment but still comparable. We did not find "perfect" discrimination for a 2-h retention interval, since the discrimination threshold more than tripled from 0.115 to 0.383 . The difference between slopes for 5-sec and 2-h ISIs turned out to be statistically significant on a $5 \%$ level (see Figures 2 and 4 ).

As is illustrated in Figure 3 the psychometric functions for individual observers with a 2-h ISI and a 22-h ITI have steeper slopes than does the function for group data with a 5-sec ISI $(n=75)$. Mean slope estimates for R.G., L.O., and M.L. are located above the $95.3 \%$ confidence intervals at $25 \%, 50 \%$, and $75 \%$ thinner responses. Significantly steeper slopes indicate that a restricted view, a 2-h ISI, and a 22-h ITI did not impair individual discrimination thresholds. Even orthogonal orientation between reference and test gratings lowered the slope for Observer L.O. only a little, corroborating similar findings on orientation independence for shorter retention intervals (e.g., Magnussen, Greenlee, Asplund, \& Dyrnes, 1990; Regan, 1985).

At the same time, the PSEs of 2.64, 3.06, and $3.38 \mathrm{c} /$ deg for Observers R.G., M.L., and L.O., respectively, are all significantly different from each other. Only Observer M.L., who was experienced in this task, has a PSE that corresponds to the reference value.

The main findings are summarized in Figure 4. Mean slope estimates increase systematically for group data with a 2-h ISI and a 5-sec ISI and for individual data with a 2-h ISI (Observer R.G.). Slope estimates for group data with 2-h and 5-sec ISIs are statistically different from each other, with mean estimates beyond the $95.3 \%$ confidence intervals. Confidence intervals for individual observers are large due to the small number of observations per data point.

\section{DISCUSSION}

Visual memory for spatial frequency appears to be more precise after $5 \mathrm{sec}$ than after $2 \mathrm{~h}$, contradicting earlier findings. This result may be interpreted as evidence for interference or decay of visual memory, illustrating, for example, the difficulty of spatial frequency information in visual short-term memory entering visual longterm memory after a single stimulus presentation (e.g., Broadbent, 1958). However, there are two reasons why a modal interpretation of the data appears insufficient. (1) It cannot explain the difference between the present 2-h ISI and Magnussen et al.'s (2003) 24-h ISI group thresholds. It seems plausible that presentation of example gratings on screen during instruction and immediately before the reference helped to establish an initial response criterion at around the reference frequency. Thus, group thresholds for spatial frequency discrimination are not simply a function of visual memory but may reflect how close together observers have established and retained individual response criteria before testing. (2) If visual memory decays over a 2-h interval, individual performance should be affected in the same way, but the slopes of the psychometric functions fitted to individual data with a 2-h ISI are steeper than the slopes for group data with 5-sec and

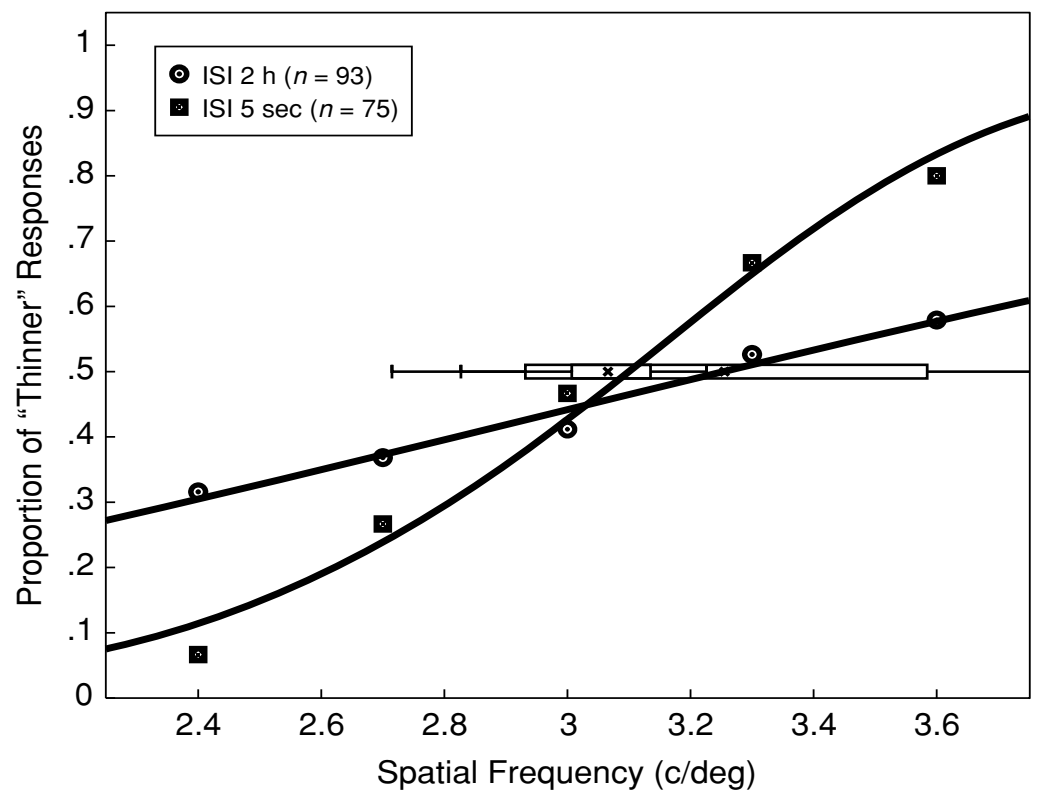

Figure 2. Weibull psychometric functions fitted to proportion of thinner responses in the present experiment. Circles depict group data $(n=93)$ for a retention interval of $2 \mathrm{~h}($ ISI $=2 \mathrm{~h})$, and squares depict group data $(n=75)$ for a retention interval of $5 \mathrm{sec}($ ISI $=5 \mathrm{sec})$. 


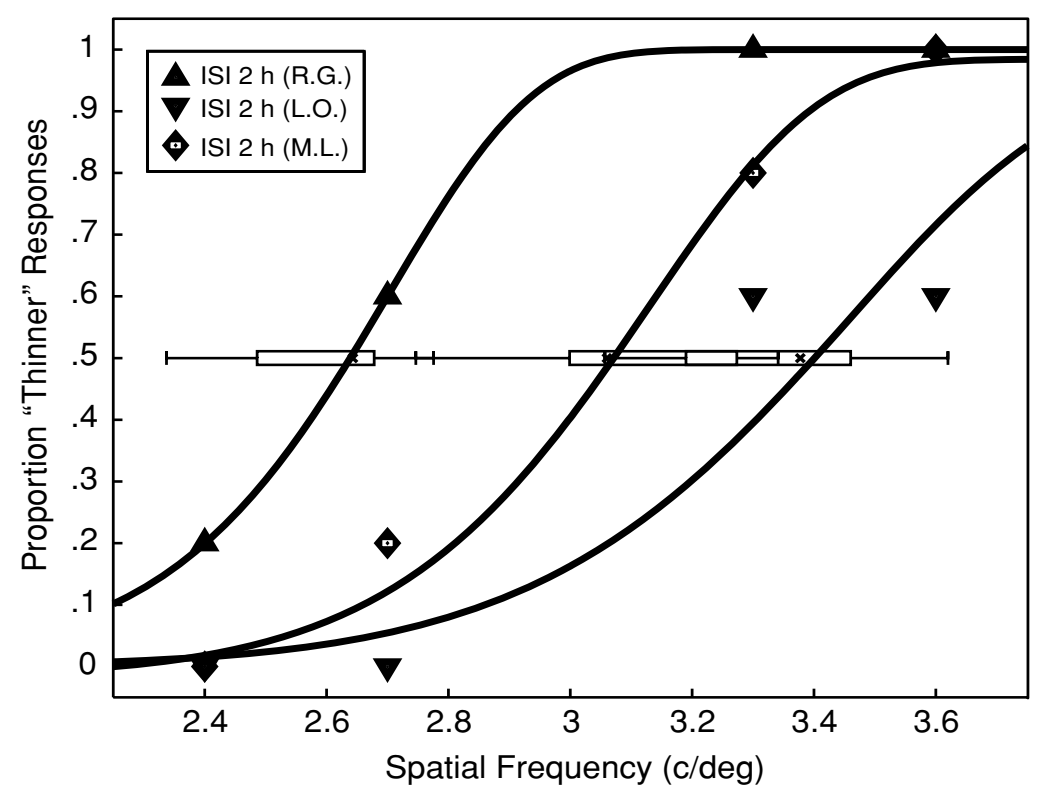

Figure 3. Weibull psychometric functions fitted to proportion of thinner responses of individual observers R.G., L.O., and M.L., with a retention interval of $2 \mathrm{~h}$ and a $22-\mathrm{h}$ intertrial interval.

2-h ISIs. Since the 22-h ITI by far exceeds the 2-h ISI, it is suggested that intertrial dependencies must be strong and long term, since they more than offset the effects of interference or decay in visual memory.
Comparing group and individual discrimination thresholds or slopes suggests better individual discrimination in repeated trials and worse group discrimination in single trials for the same 2-h retention interval. Both results

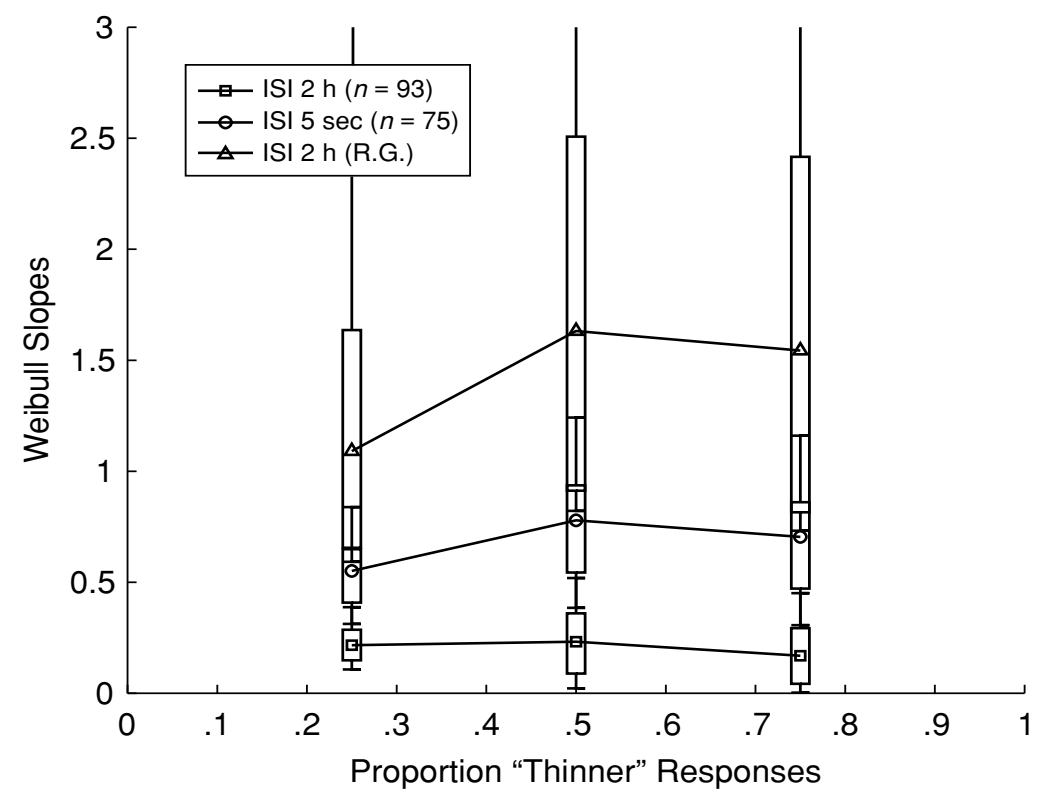

Figure 4. Comparison of estimated slopes for group data (squares, ISI $=\mathbf{2} \mathbf{~ h}$, $n=93$; circles, ISI $=5 \mathrm{sec}, \boldsymbol{n}=75$ ) and individual data (triangles, R.G., ISI = $2 \mathrm{~h})$. Slopes are first derivatives of fitted Weibull functions at $25 \%, 50 \%$, and $75 \%$ points. Confidence intervals are obtained by bootstrapping (Wichmann \& Hill, 2001b). Boxes and bars indicate Weibull confidence intervals of $68.3 \%$ and $95.3 \%$, respectively. 
are compatible with the idea that uncertainty about the reference affects discrimination performance but do not support the assumption of a "perfect" visual long-term memory for spatial frequency.

Finally, comparing Magnussen et al.'s (2003) results with our present results shows a slightly raised discrimination threshold for 5-sec ISI group data but a four-fold increase for 2-h ISI group data. In our view, these changes are effects of differing instructions and uncertainty about the reference. After a 5-sec interval, it seems plausible that visual memory of the reference still dominates discrimination, but after a 2-h delay, visual memory of the reference seems to be weak, if not absent.

Although plausible, our explanation in terms of criterion setting rests on indirect evidence. Estimates of the PSEs and their confidence intervals provide only a rough approximation, and larger data sets are usually required to assess criterion setting in a discrimination task (e.g., Lages, 1998). In the framework of classical SDT, it is possible to measure a criterion by repeatedly presenting the reference stimulus as a test stimulus (e.g., Green \& Swets, 1974; Wickens, 2002). If we estimate the response criterion by $-z$ (false alarm), where proportion of thinner responses at the reference value serves as the false alarm rate, a criterion of 0.09 for 5 -sec ISI and 0.22 for 2-h ISI group data confirms our assumption that in the present discrimination task, PSE shifts with the response criterion.

There are two reasons why we did not measure a response criterion for individual data. In the context of "perfect" visual memory, repeated presentation of the reference as the test stimulus may lead to identification, inviting guessing strategies in forced choice. In the framework of criterion setting, repeated presentation of the reference as the test stimulus might help to adjust the criterion to the reference value.

\section{CONCLUSION}

The present results show a significant difference between 5-sec and 2-h ISI group discrimination and a significant increase of individual discrimination based on repeated trials, in comparison with group discrimination based on single trials. Significant shifts of the PSE for group and individual data seem to indicate uncertainty about the reference and the presence of criterion setting, but this interpretation needs to be confirmed. Uncertainty about the reference in single test trials would also explain the increase of the group discrimination threshold, but we cannot rule out interference or decay of visual memory. Evidence from carefully conducted experiments on rapid decay in visual short-term memory (Gold, Murray, Sekuler, Bennett, \& Sekuler, 2005; Harvey, 1986; Laming $\&$ Scheiwiller, 1985) supports our view that task-relevant information, rather than sensory information in a "perfect" visual long-term memory, persists over time.

Natural scenes are rich in spatial frequency content, and a single spatial frequency rarely captures an entire object or scene. If the visual system stores low-level information of objects over prolonged periods of time, it would need to retain a wide range of spatial frequency information, together with other low-level information (e.g., orientation, contrast, or color), to establish a long-term memory of a single object or scene. Consider spatial frequency discrimination for stimuli that are slightly more complex than a sine wave grating. If observers had to remember a square wave in a discrimination task and did not retain a response criterion for the width of the bars, they would need to extract and recall the spatial frequency of the fundamental. If the stimulus were a square wave with missing fundamental, they would even need to infer the spatial frequency of the missing fundamental from higher harmonics. Reasonable discrimination performance for these stimuli would indicate that in a delayed discrimination task, observers are likely to retain a response criterion for stimulus size, rather than low-level spatial frequency information.

On the basis of psychophysical and neurophysiological evidence, Magnussen (2000) and Magnussen and Greenlee (1999) proposed that a low-level perceptual memory system is located at a site beyond V1 but prior to a structural description system of the perceptual representation system (Tulving \& Schacter, 1990). Although the type of information stored there is supposed to be low level, the sensory memory system for spatial frequency would be located at a stage at which other stimulus dimensions, such as orientation, have no effect on memory-masking and dual-judgment tasks. Instead, it has been shown that the effects of memory masking are task dependent (Lalonde \& Chaudhuri, 2002) and that independence of orientation in a spatial frequency discrimination task and independence of spatial frequency in an orientation discrimination task are likely to be by-products of processes at the decision stage (Lages \& Treisman, 1998, 2006). These findings point to the involvement of a long-term memory for decision processes, rather than a sensory long-term memory for low-level spatial information.

It is generally acknowledged that spatial frequency filters serve the purpose of optimally encoding local spatial information, irrespective of image content (Daugman, 1980; De Valois \& De Valois, 1988). Spatial frequency is, by definition, independent of viewing distance, orientation, and phase. What is the evolutionary advantage of a dedicated visual long-term memory for information that is not scaled in size and position? Magnussen et al. (2003) argued that visual memory for spatial frequency may assist in the formation of more permanent highprecision representations of objects and meaningful patterns. But the same spatial frequency information can appear in arbitrary objects and patterns at different positions and viewing distances. Therefore, without additional depth information, spatial frequency carries limited objectspecific information.

The setting of a decision criterion, on the other hand, is a useful and simple strategy for optimizing performance in a discrimination task. A response criterion can relate to arbitrary dimensions of visual or other sensory input, and it is stored, updated, and retrieved with considerable ease from short- or long-term memory. 
On the basis of the present results and discussion, it can be concluded that the concept of a "perfect" visual long-term memory dedicated to spatial frequency and other low-level attributes requires additional verification. The use of sine wave gratings with low-level attributes in a psychophysical discrimination task does not automatically safeguard performance against high-level processing at the decision stage.

\section{REFERENCES}

Ashby, F. G., Maddox, W. T., \& Lee, W. W. (1994). On the dangers of averaging across subjects when using multidimensional scaling or the similarity choice model. Psychological Science, 5, 144-151.

Broadbent, D. E. (1958). Perception and communication. New York: Pergamon.

Cowan, N. (1999). An embedded-processes model of working memory. In A. Miyake \& P. Shah (Eds.), Models of working memory: Mechanisms of active maintenance and executive control (pp. 62-101). New York: Cambridge University Press.

Crowder, R. G. (1993). Systems and principles in memory theory: Another critique of pure memory. In A. F. Collins, S. E. Gathercole, M. A. Conway, \& P. E. Morris (Eds.), Theories of memory (pp. 139161). Hove, U.K.: Erlbaum.

Daugman, J. G. (1980). Two-dimensional spectral analysis of cortical receptive field profiles. Vision Research, 20, 847-856.

De Valois, R. L, \& De Valois, K. K. (1988). Spatial vision. New York: Oxford University Press.

Gold, J. M., Murray, R. F., Sekuler, A. B., Bennett, P. J., \& SeKuler, R. (2005). Visual memory decay is deterministic. Psychological Science, 16, 769-774.

Green, D. M., \& Swets, J. W. (1974). Signal detection theory and psychophysics. New York: Krieger.

Harvey, L. D. (1986). Visual memory: What is remembered? In F. Klix \& H. Hagendorf (Eds.), Human memory and cognitive capabilities (Vol. 1, pp. 173-187). Amsterdam: North-Holland.

LAGES, M. (1998). Bias in visual discrimination and detection. Unpublished doctoral dissertation, Oxford University.

Lages, M., \& Treisman, M. (1998). Spatial frequency discrimination: Visual long-term memory or criterion setting? Vision Research, 38, 557-572.

Lages, M., \& Treisman, M. (2006). Orientation discrimination and criterion setting. Manuscript in preparation.

Lalonde, J., \& Chaudhuri, A. (2002). Task-dependent transfer of perceptual to memory representations during delayed spatial frequency discrimination. Vision Research, 42, 1759-1769.

LAming, D., \& Scheiwiller, P. (1985). Retention in perceptual memory: A review of models and data. Perception \& Psychophysics, 37, 189-197.

Magnussen, S. (2000). Low-level memory processes in vision. Trends in Neurosciences, 6, 247-251.
Magnussen, S., \& Dyrnes, S. (1994). High-fidelity perceptual longterm memory. Psychological Science, 5, 99-102.

Magnussen, S., \& Greenlee, M. W. (1999). The psychophysics of perceptual memory. Psychological Research, 62, 81-92.

Magnussen, S., Greenlee, M. W., Aslaksen, P. M., \& Kildebo, O. O. (2003). High-fidelity perceptual long-term memory revisited-and confirmed. Psychological Science, 14, 74-76.

Magnussen, S., Greenlee, M. W., Asplund, R., \& Dyrnes, S. (1990). Perfect short-term memory for periodic patterns. European Journal of Cognitive Psychology, 2, 245-262.

NAIRNE, J. S. (1990). A feature model of immediate memory. Memory \& Cognition, 18, 251-269.

NAIRNE, J. S. (2003). Sensory and working memory. In I. B. Weiner (Series Ed.) \& A. H. Healy \& R. W. Proctor (Vol. Eds.), Handbook of psychology: Vol. 4. Experimental psychology (pp. 423-444). New York: Wiley.

REGAN, D. (1985). Storage of spatial-frequency information and spatialfrequency discrimination. Journal of the Optical Society of America A, 2, 619-621.

Rensink, R. A., O'Regan, J. K., \& Clark, J. J. (2000). On the failure to detect changes in scenes across brief interruptions. Visual Cognition, 7, 127-145.

Rock, I., \& ENGELSTEIN, P. (1959). A study of memory for visual form. American Journal of Psychology, 72, 221-229.

STANDING, L. (1973). Learning 10,000 pictures. Quarterly Journal of Experimental Psychology, 25, 207-222.

SwifT, D. J., \& Smith, R. A. (1983). Spatial frequency masking and Weber's law. Vision Research, 23, 495-505.

Treisman, M., \& Faulkner, A. (1985). Can decision criteria interchange locations? Some positive evidence. Journal of Experimental Psychology: Human Perception \& Performance, 11, 187-208.

Treisman, M., \& Williams, T. C. (1984). A theory of criterion setting with an application to sequential dependencies. Psychological Review, 91, 68-111.

Tulving, E., \& Schacter, D. L. (1990). Priming and human memory systems. Science, 247, 301-306.

Vogels, R., \& Orban, G. A. (1986). Decision factors affecting line orientation judgments in the method of single stimuli. Perception \& Psychophysics, 40, 74-84.

Wenger, M. J, \& RASChE, C. (2005). Bias in an unbiased land? Criterion shifts in perceptual learning using two-interval two-alternative forcedchoice staircase procedure [Abstract]. Journal of Vision, 5, 867a.

Wichmann, F. A., \& HiLl, N. J. (2001a). The psychometric function: I. Fitting, sampling, and goodness of fit. Perception \& Psychophysics, 63, 1293-1313.

WichmanN, F. A., \& Hill, N. J. (2001b). The psychometric function: II. Bootstrap-based confidence intervals and sampling. Perception \& Psychophysics, 63, 1314-1329.

WiCKEnS, T. D. (2002). Elementary signal detection theory. Oxford: Oxford University Press.

(Manuscript received December 16, 2004; revision accepted for publication October 11, 2005.) 